\title{
Touch-based Fingertip Blood-Free Reliable Glucose Monitoring: Personalized Data Processing for Predicting Blood Glucose Concentrations
}

Juliane R. Sempionatto, ${ }^{\dagger}$ Jong-Min Moon,$^{\dagger}$ and Joseph Wang*,

Dept Nanoengineering, University of California San Diego, La Jolla, CA 92093, USA

†These authors contributed equally to this work

*Corresponding author: josephwang@eng.ucsd.edu 


\section{Supporting Information}
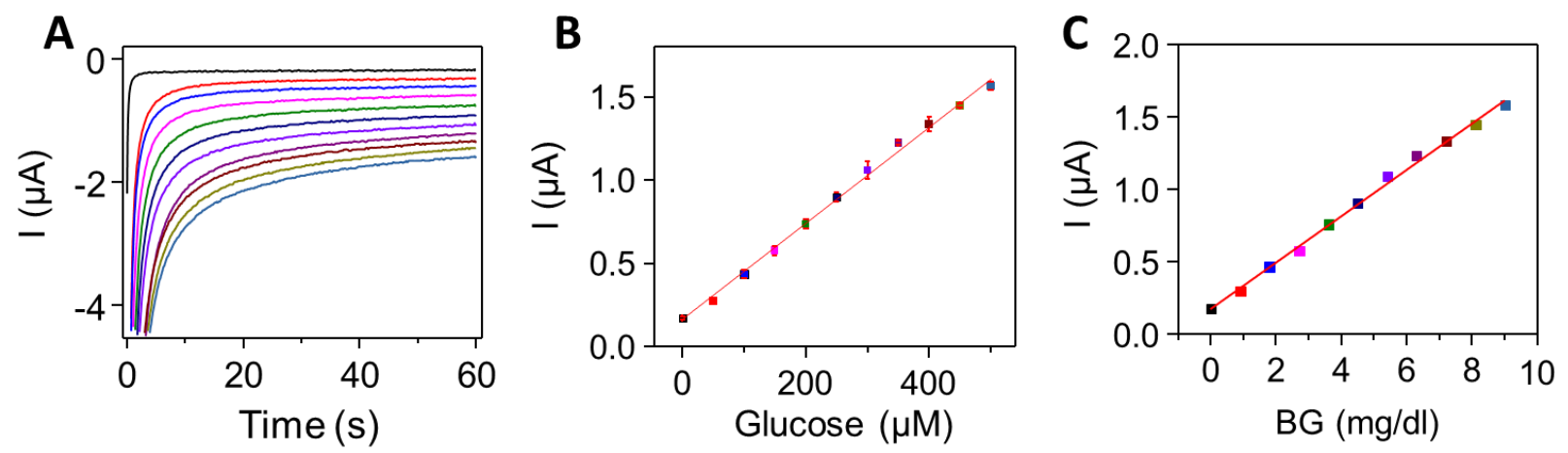

Figure S1. In vitro calibration curve for the glucose sensor. A) Chronoamperogram for successive additions of $50 \mu \mathrm{M}$ of glucose in $0.1 \mathrm{M}$ PBS pH 7 at $-0.2 \mathrm{~V}$ for 60 seconds. B) Calibration plot obtained from these chronoamperograms (RSD 6.2\%, $\mathrm{n}=3$ ). C) Calibration plot of current signal vs blood glucose in $\mathrm{mg} / \mathrm{dl}$. 

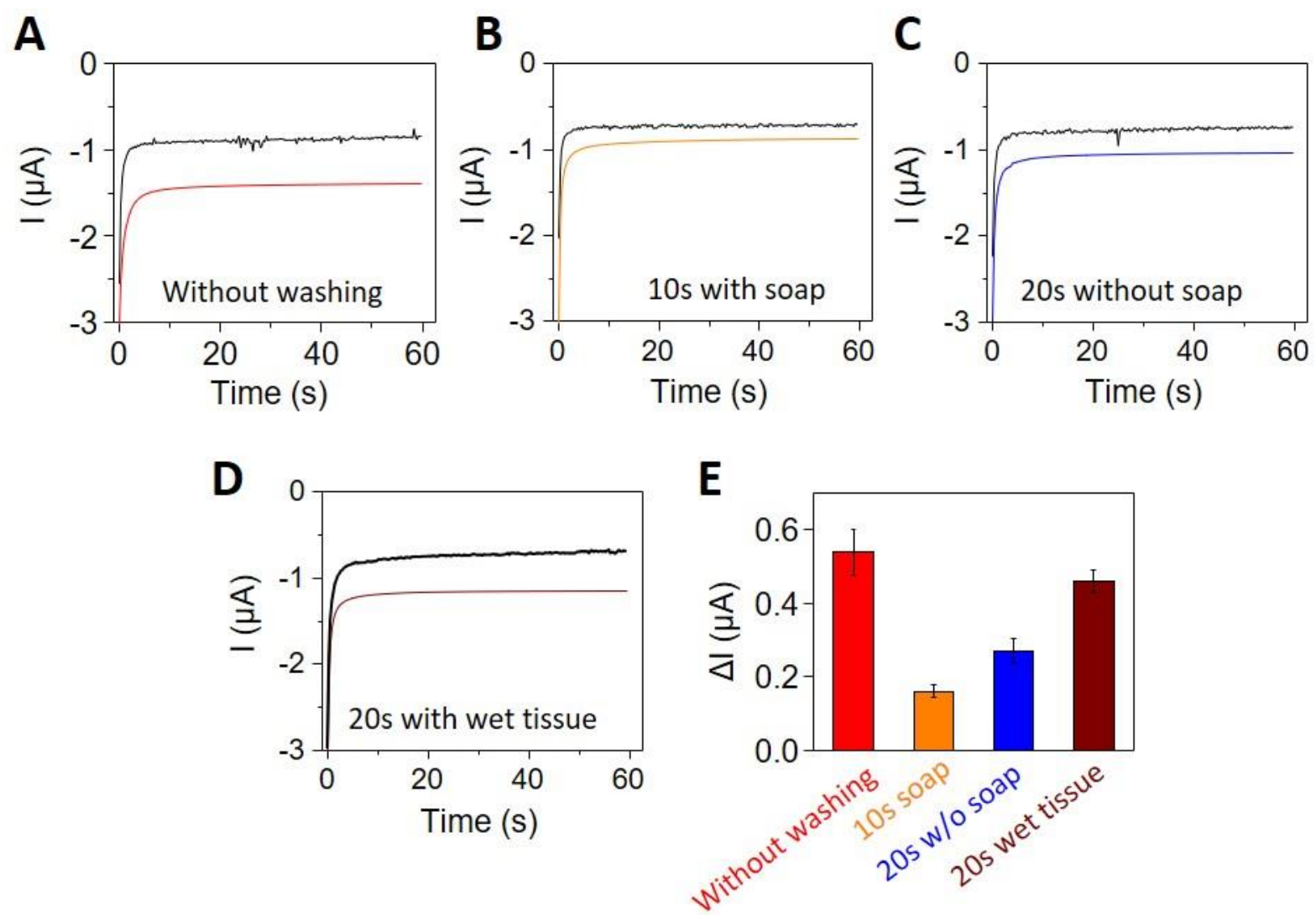

Figure S2. Optimization of the hand washing step with three repeated experiments using different glucose sensor. A) Without washing hands. B) $10 \mathrm{~s}$ washing with soap. C) $20 \mathrm{~s}$ washing without soap. D) $20 \mathrm{~s}$ continuous swiping with wet tissue. E) The bar plots for the optimization of the hand washing step. 

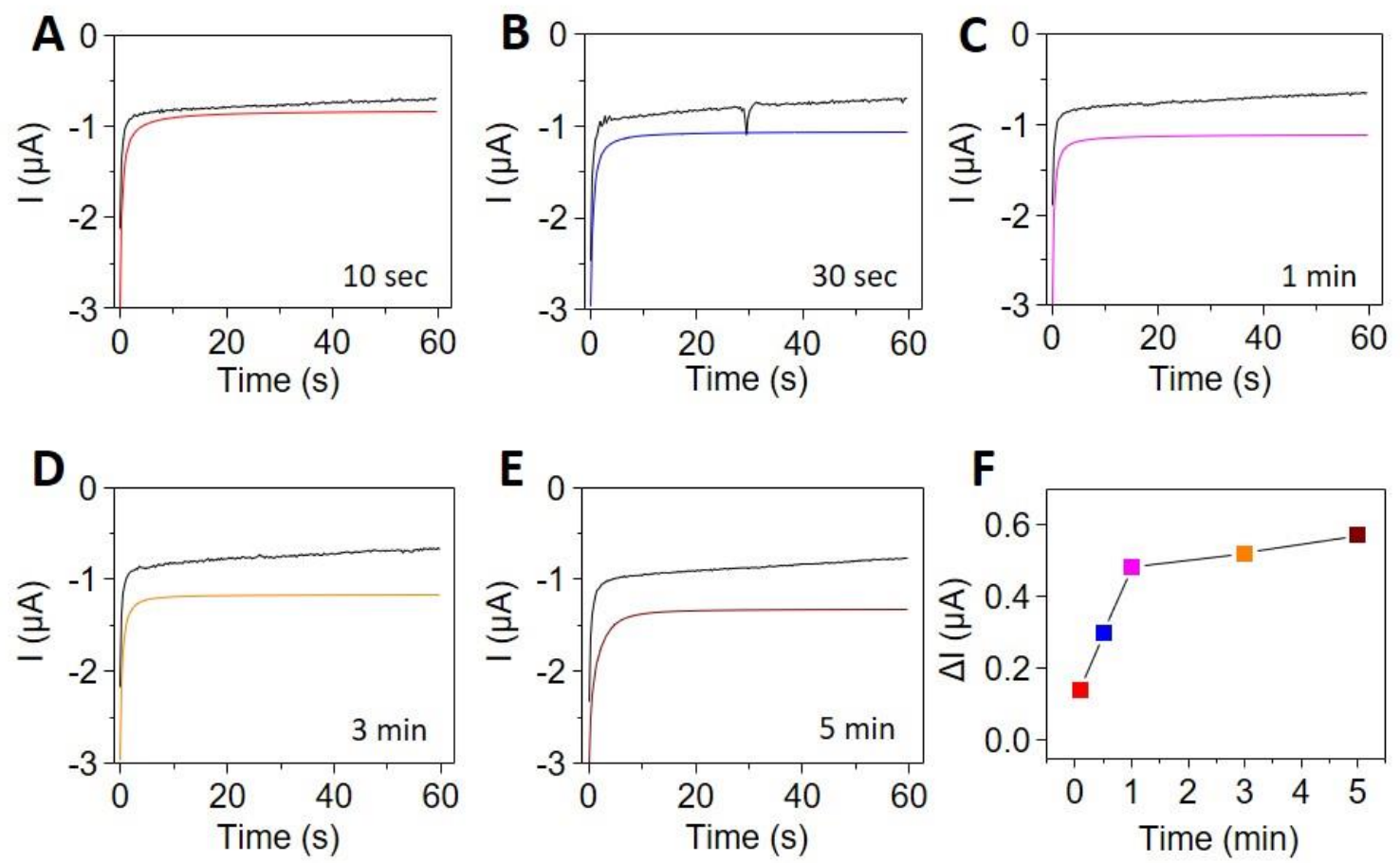

Figure S3. Optimization of the touching time. Touching the sensor for A) $10 \mathrm{sec}$, B) $30 \mathrm{sec}$, C) $1 \mathrm{~min}$, D) 3 min, and E) 5 min. F) Response vs. touching time plot: optimal touching time is 1 minute. 
A

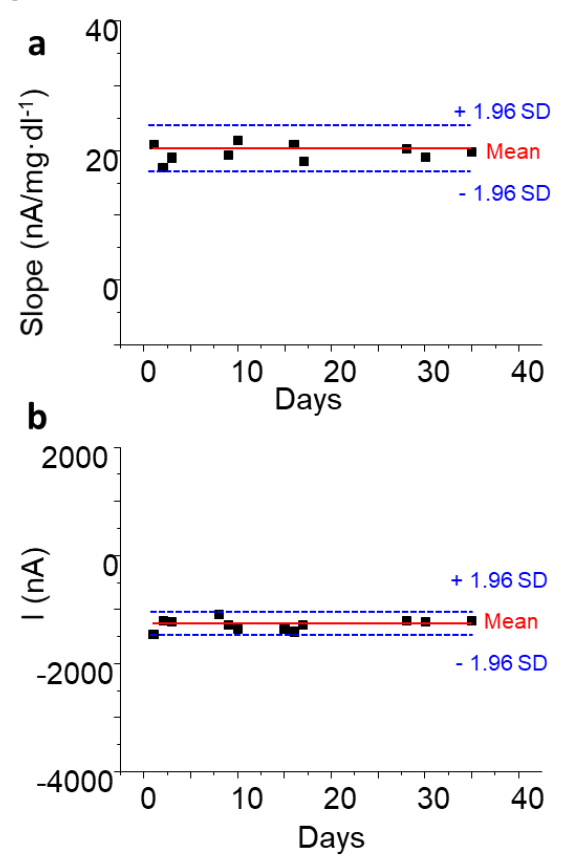

B a
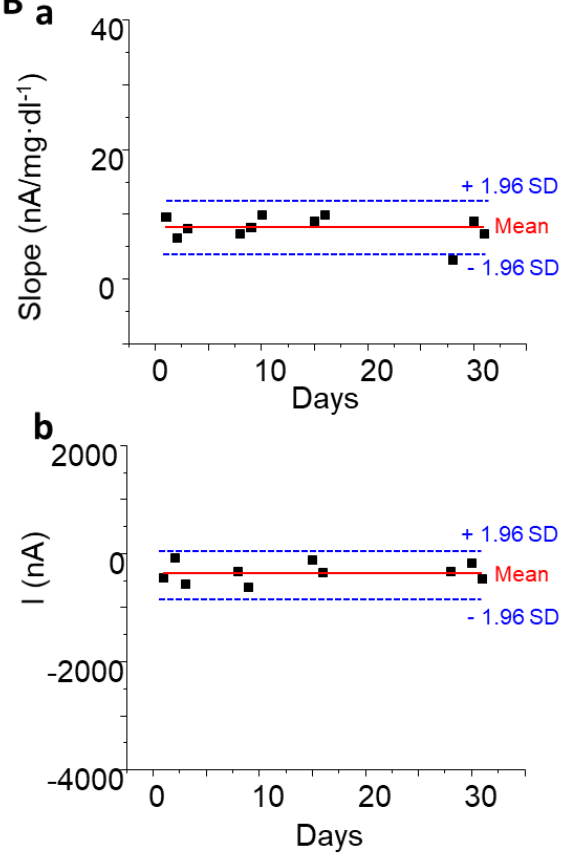

Figure S4. Stability of the personal factors, slope (a) and intercept (b) for two subjects (A, B) over a 4 weeks period with confidence bands of $95 \%$. 


\section{Rationing for the personalized calibration software}

The initial calibration is acquired using equations 1-3 and the sweat personal values are computed in the software where the corresponding blood glucose concentration is calculated. Once or twice a month, a new validated data would be inserted in the software for the moving average calculation. This new inserted value is first validated (e.g. outlier detection) and if the value is within the expected range, it can be included in the initial calculated calibration curve to obtain the new averaged parameters. If the inserted blood value is outside the confidence interval, the value is rejected, and a new input is requested. If the software rejects three values consecutively, the software indicates the need of a whole new calibration plot (Figure S5). This protocol ensures automation of the personal mathematical treatment and high quality for the output values. The friendly software was well accepted by the volunteers, showing good performance and readout values.

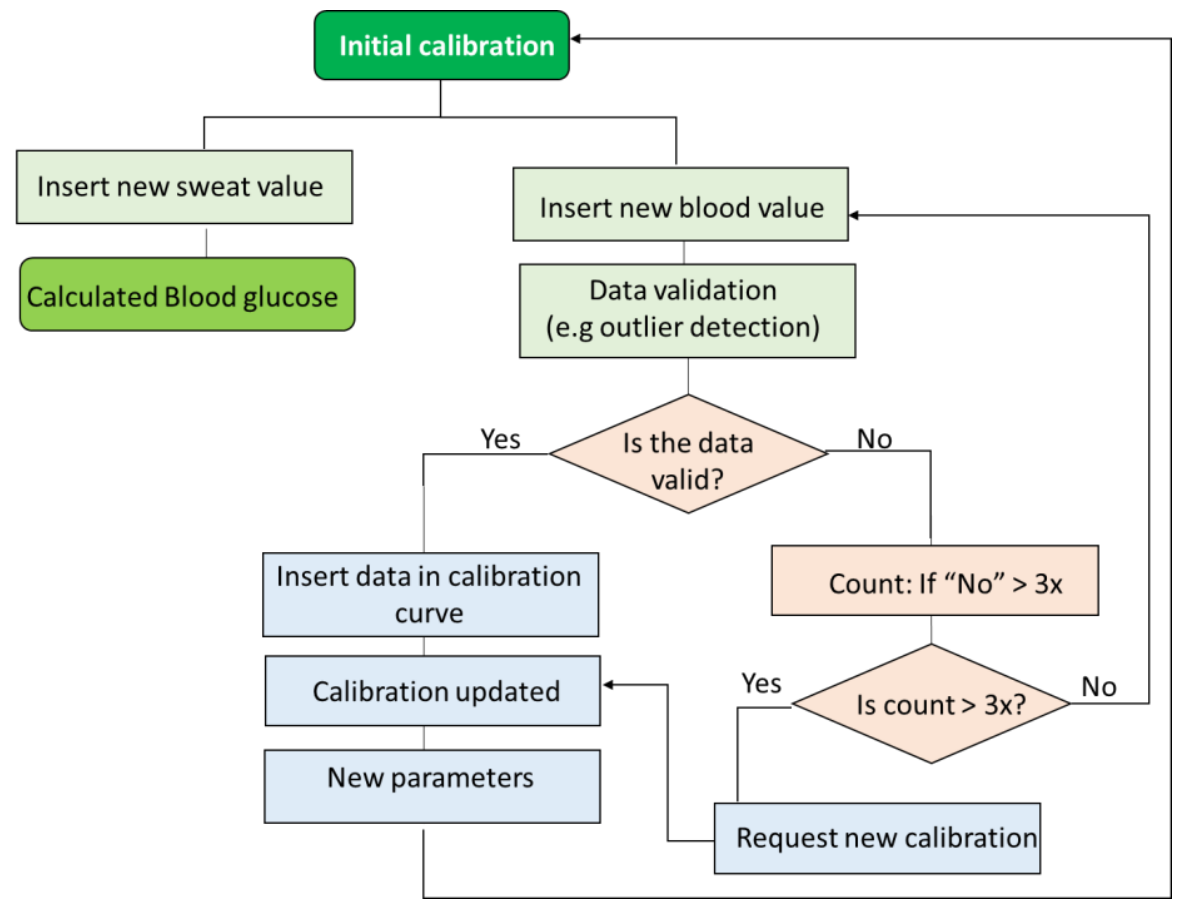

Figure S5. Flowchart for the calibration and analysis of sweat glucose signals to blood glucose concentrations using the fingertip touch-based sensor. 


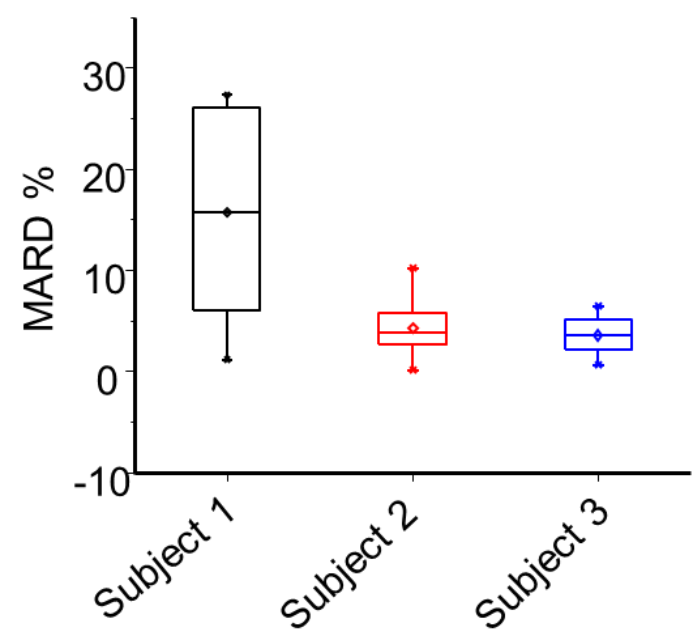

Figure S6. Box plots for the MARD on successive study measurements during the day for three subjects. Displayed are mean (diamonds), median (horizontal lines within boxes), 25th and 75th percentiles (lower and upper edge of the boxes), and minimum and maximum values (Whiskers). 